\title{
Evaluación cardiovascular del potencial receptor de trasplante renal
}

\section{Cardiovascular evaluation of potential kidney transplant recipient}

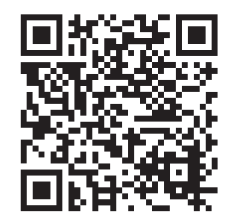

\author{
Abraham Cohen-Bucay* \\ * Nefrólogo de trasplante. Massachusetts General Hospital. \\ Adjunto de Medicina. Massachusetts General Hospital. \\ Instructor de Medicina. Harvard Medical School.
}

Todos los potenciales receptores de trasplante renal deben tener una evaluación cardiovascular con el propósito de disminuir los eventos y mortalidad cardiovascular en el periodo perioperatorio y a largo plazo basada en historia clínica, exploración física y electrocardiograma.

La enfermedad renal crónica es un factor de riesgo independiente de enfermedad coronaria dado que la incidencia y severidad de enfermedad coronaria aumenta conforme la tasa de filtrado glomerular disminuye. ${ }^{1,2}$ En pacientes asintomáticos con enfermedad renal crónica terminal (ERCT), la prevalencia de estenosis arterial coronaria es de 37 a $58 \% .^{3}$ En el periodo postrasplante, se le atribuye $30 \%$ de las muertes a enfermedad cardiovascular, siendo así la principal causa de muerte en receptores de trasplante renal. ${ }^{4}$ La incidencia acumulada de infarto a miocardio dentro de los tres años después del trasplante es de 4.2 a $11.1 \% .^{5,6}$ Ya que la incidencia/prevalencia, así como la morbilidad y mortalidad asociada con enfermedad cardiovascular es tan elevada en los pacientes con enfermedad renal crónica terminal y en receptores de trasplante renal, es necesario realizar una evaluación cardiovascular en todos los potenciales receptores.

Es importante notar que la evaluación cardiovascular del potencial receptor no debe ser la misma evalua- ción que para cualquier otra cirugía no cardiaca. Esto debido a que un trasplante renal hace uso de un recurso muy limitado que se debe destinar a los pacientes que tengan proyectada una buena supervivencia posterior al trasplante. Además, como ya se mencionó anteriormente, los pacientes que requieren un trasplante renal representan una población de alto riesgo cardiovascular. Por último, receptores de trasplante renal que requieren una intervención cardiovascular como revascularización endovenosa o cirugía cardiovascular, tienen un alto índice de disfunción severa o incluso pérdida del injerto. Se ha encontrado que 6-33\% de los pacientes que requieren cirugía cardiovascular después del trasplante presentan disfunción severa del injerto y $3-12 \%$ pierden el injerto. ${ }^{7-10}$ Por estos motivos, el propósito de la evaluación cardiovascular no se debe limitar a disminuir la mortalidad y eventos cardiovasculares en el periodo peritrasplante, sino que también lo debe hacer a largo plazo.

Los pacientes con síntomas de enfermedad cardiovascular activa deben ser evaluados por cardiología.

Aunque no es absolutamente necesario que cardiología evalúe a todos los potenciales receptores de trasplante renal, la población con síntomas cardiovasculares sí deben ser evaluados por un cardiólogo con historia clínica, exploración física y los estudios de ga- 
binete que considere necesarios para descartar que el paciente tenga enfermedad cardiovascular activa que requiera tratamiento y que contraindique en ese momento el trasplante.

Con base en los factores de riesgo del paciente se deberá categorizar como de bajo, intermedio o alto riesgo cardiovascular, y se deberán hacer los estudios necesarios según la Tabla 1.

Los pacientes con enfermedad renal crónica son una población muy heterogénea con diferentes factores de riesgo cardiovasculares, por lo que la evaluación cardiovascular se debe individualizar. Los factores de riesgo cardiovasculares en esta población son:4,11

- Enfermedad ateroesclerótica previa: incluyendo enfermedad coronaria, enfermedad cerebrovascular y enfermedad arterial periférica.

- Diabetes mellitus.

- Enfermedad renal crónica terminal con requerimiento de terapia de reemplazo renal por más de un año.

- Hipertrofia de ventrículo izquierdo.

- Edad mayor de 60 años.

- Tabaquismo previo o actual.
- Hipertensión arterial sistémica.

- Dislipidemia.

Se ha propuesto clasificar a esta población en grupos de riesgo con base en síntomas, capacidad funcional, scores de enfermedad cardiovascular o número de factores de riesgo. Dado que de 37 a $58 \%$ de los pacientes con ERCT asintomáticos tienen estenosis coronaria, ${ }^{3}$ la evaluación cardiovascular no se debe basar en síntomas ni capacidad funcional. El score que más se ha usado en la población general para evaluar el riesgo cardiovascular es el que se basa en el estudio de Framingham; sin embargo, en pacientes que requieren trasplante renal, el score de Framingham infraestima el riesgo significativamente. ${ }^{6,12,13}$ Por lo tanto, no se recomienda el uso del score de Framingham para valoración de riesgo cardiovascular previo a trasplante renal.

Debido a que el riesgo cardiovascular conferido por cada factor es diferente, no consideramos que una simple sumatoria de factores de riesgo sea apropiada. Los dos factores que confieren mayor riesgo son diabetes y enfermedad coronaria previa.4-6 Por esta razón, en muchas otras situaciones clínicas se considera a la diabetes como un equivalente de enfermedad coronaria y por lo mismo, consideramos a

Tabla 1: Estudios requeridos para la evaluación cardiovascular acorde a su clasificación de riesgo en el potencial receptor de trasplante renal.

\begin{tabular}{|c|c|c|c|}
\hline Categoría de riesgo & Factores de riesgo & Estudios requeridos & Intervalo de tiempo \\
\hline Bajo & $\begin{array}{l}\text { - Sin angina } \\
\text { - Sin enfermedad cardiovascular } \\
\text { - Sin diabetes } \\
\text { - Sin enfermedad vascular periférica } \\
\text { - } \leq 2 \text { factores de riesgo: } \\
\text { - Edad > } 60 \\
\text { - Hipertensión } \\
\text { - Dislipidemia } \\
\text { - Diálisis > } 1 \text { año } \\
\text { - Tabaquismo } \\
\text { - Hipertrofia de ventrículo izquierdo } \\
\text { - Historia familiar muerte cardiovascular temprana }\end{array}$ & Ecocardiograma en reposo & Repetir cada 2 años \\
\hline Intermedio & $\begin{array}{l}\text { - Sin angina } \\
\text { - Sin enfermedad cardiovascular } \\
\text { - Riesgo cardiovascular intermedio: } \\
\text { - DM sin microangiopatía, } 0 \\
- \text { Enfermedad vascular periférica, } 0 \\
-\geq 3 \text { factores de riesgo }\end{array}$ & $\begin{array}{c}\text { Prueba de esfuerzo (ecocardiograma } \\
\text { con dobutamina o estudio nuclear) } \\
++\underset{+}{\text { Ecocardiograma en reposo }}\end{array}$ & Repetir cada año \\
\hline Alto & $\begin{array}{l}\text { - DM con microangiopatía } \\
\text { - Enfermedad cardiovascular previa } \\
\text { - Síntomas de enfermedad coronaria (angina) }\end{array}$ & $\begin{array}{c}\text { Coronariografía } \\
+ \\
\text { Ecocardiograma en reposo }\end{array}$ & Repetir cada 6 a 12 meses \\
\hline
\end{tabular}


Tabla 2: Rendimiento de pruebas diagnósticas cardiovasculares en el potencial receptor de trasplante renal.

\begin{tabular}{lcc}
\hline \multicolumn{1}{c}{ Estudio } & Sensibilidad & Especificidad \\
\hline $\begin{array}{l}\text { Ecocardiograma de esfuerzo } \\
\text { con dobutamina }^{14}\end{array}$ & $0.79(0.67-0.88)$ & $0.89(0.81-0.94)$ \\
$\begin{array}{l}\text { Estudio nuclear de perfusión } \\
\text { miocárdica }^{14}\end{array}$ & $0.74(0.54-0.87)$ & $0.70(0.51-0.84)$ \\
\hline
\end{tabular}

pacientes con diabetes con daño microvascular (diabetes como causa de ERCT, retinopatía y/o neuropatía) como de alto riesgo cardiovascular, al igual que aquéllos con enfermedad cardiovascular previa y los pacientes con síntomas de enfermedad cardiovascular activa. Los pacientes sin estas características, sin enfermedad vascular periférica y dos o menos factores de riesgo son considerados como riesgo cardiovascular bajo, mientras que aquellos pacientes con más de dos factores de riesgo, enfermedad vascular periférica o diabetes sin evidencia de microangiopatía se consideran como riesgo cardiovascular intermedio.

La decisión de qué estudios realizar en cada paciente se debe basar en la sensibilidad y especificidad de dichos estudios en la población de pacientes con ERCT de acuerdo con la siguiente Tabla 2.

También se debe considerar que dependiendo de la probabilidad preprueba (grupo de riesgo cardiovascular) cambia la probabilidad postprueba del estudio realizado. En pacientes de bajo riesgo hay una alta incidencia de falsos positivos, mientras que en pacientes de alto riesgo hay una gran incidencia de falsos negativos y el mayor valor de las pruebas no invasivas cardiovasculares es en el grupo de riesgo intermedio. ${ }^{14}$ Con base en esta información, recomendamos el uso de pruebas no invasivas solamente en el grupo de riesgo intermedio. En el grupo de riesgo cardiovascular bajo no se requiere mayor evaluación coronaria, mientras que el grupo de riesgo cardiovascular alto requiere de evaluación por cardiología y coronariografía.

En todos los grupos de riesgo se recomienda realizar un ecocardiograma en reposo, ya que como se menciona posteriormente, las lesiones estructurales cardiacas (FEVI disminuidas, enfermedad valvular severa, hipertensión pulmonar) se consideran contraindicaciones de trasplante renal.

El intervalo de tiempo en que se tienen que repetir los estudios también cambia dependiendo el grupo de riesgo; sin embargo, no hay estudios de buena calidad que nos informen el intervalo de tiempo óptimo y esta recomendación está basada en la opinión de los autores de esta guía.

El uso de score de calcio y tomografía coronaria no se recomienda como método de evaluación pretrasplante.

Se han realizado hasta la fecha pocos estudios que evalúan los estudios no invasivos de anatomía coronaria en la población de pacientes con ERCT..$^{15-19}$ Además, dichos estudios han mostrado resultados discordantes. ${ }^{15-18}$ Por esta razón, no recomendamos el uso de score de calcio o tomografía coronaria en esta población (Tabla 3).

Aquellos pacientes que tengan

prueba de esfuerzo positiva, deberán

estudiarse con coronariografía.

En un metaanálisis ${ }^{20}$ no se demostró que la coronariografía es superior a las pruebas no invasivas en la predicción de eventos cardiovasculares mayores posterior al trasplante; sin embargo, la habilidad de la coronariografía para predecir eventos cardiovasculares estaba falsamente reducida por la revascularización realizada durante el mismo procedimiento. Además, los pacientes referidos para coronariografía seguramente también tenían una prueba no invasiva anormal previo a la coronariografía, lo cual sesga

Tabla 3: Rendimiento de nuevas pruebas diagnósticas cardiovasculares.

\begin{tabular}{lccc}
\hline \multicolumn{1}{c}{ Estudio } & Sensibilidad & Especificidad & Observaciones \\
\hline CACS $^{15-18}$ & $0.54-0.92$ & $0.44-0.87$ & 4 estudios, $n$ varía entre 18 y 148. Total $n=309$. Se usaron diferentes valores en cada estudio \\
Tomografía coronariati,19 & $0.65-0.80$ & $0.74-0.86$ & $\begin{array}{l}2 \text { estudios, } n \text { varía entre 19 a 147. Total } n=147 \text {. El estudio más grande reportó la sensibilidad } \\
\text { y especificidad combinando tomografía coronaria con tres factores de riesgo cardiovascular }\end{array}$ \\
\hline
\end{tabular}

CACS $=$ score de calcio en arterias coronarias. 
los resultados de forma importante. Dado que todo paciente que tenga una prueba de esfuerzo positiva inmediatamente es considerado de alto riesgo cardiovascular, creemos que es necesario que dichos pacientes sean referidos a cardiología para mayor estudio con coronariografía.

Aquellos pacientes con estenosis significativa en la coronariografía deberán ser revascularizados previo al trasplante.

Una coronariografía anormal en el receptor potencial de trasplante renal confiere mayor riesgo de mortalidad (OR 2.96; IC95\% 1.25-7.00) y eventos cardiovasculares mayores (OR 16.02; IC95\% 2.42-105.98) en el periodo postrasplante,${ }^{20}$ mientras que la revascularización disminuye la mortalidad (OR 0.28; IC95\% 0.12-0.64) y los eventos cardiovasculares mayores (OR 0.19; IC95\% 0.05-0.72) en el periodo postrasplante. Por esta razón recomendamos revascularizar a todo paciente con estenosis significativa en la coronariografía previo al trasplante.

Los pacientes con fracción de eyección del ventrículo izquierdo (FEVI) menor de 30 deberán ser evaluados por cardiología en búsqueda de causas reversibles, y es considerada una contraindicación relativa para trasplante renal.

Los pacientes con enfermedad renal crónica terminal frecuentemente tienen FEVI disminuidas. En estudios japoneses se ha visto que entre 13 y $34 \%$ de los pacientes en diálisis tienen $\mathrm{FEVI}<50 \%{ }^{21,22}$ Sin embargo, estos datos no son generalizables a la población mexicana y se estima que la incidencia de FEVI disminuida en pacientes mexicanos con ERCT es mayor. Para pacientes que permanecen en lista de espera, la mortalidad es mayor en aquellos que tienen FEVI disminuida. ${ }^{21,22}$ No obstante, la FEVI puede mejorar en el periodo postrasplante. En un estudio en el que se incluyeron 103 pacientes con FEVI $<40,70 \%$ mejoró su FEVI en el postrasplante a $>50$, $15 \%$ mejoró la $\mathrm{FEVI}$ a $>40$, pero $<50$ y $15 \%$ permaneció con $\mathrm{FEVI}<40 .{ }^{23}$ La mortalidad en aquellos pacientes que permanecieron con FEVI baja ( 40 o entre 40 y 50 ) tuvieron mayor mortalidad a 50 meses postrasplante. ${ }^{23}$ El único predictor de mejoría de FEVI postrasplante fue el tiempo en diálisis (14 meses en el grupo que mejoró la FEVI vs. 40 meses en el grupo que permaneció con $\mathrm{FEVI}$ baja). ${ }^{23}$ Es por estos datos que la $\mathrm{FEVI}<30$ no se considera una contraindicación absoluta de trasplante renal. Sin embargo, recomendamos evaluación por cardiología de todos los pacientes con $\mathrm{FEVI}<30$ para iden- tificar y tratar causas reversibles de FEVI disminuida. Es importante notar que aquellos pacientes con $\mathrm{FEVI}<30$ que reciben una adecuada diálisis, que no tienen una causa reversible de FEVI disminuida, y que permanecen en diálisis por más de dos años, tienen una baja probabilidad de mejorar su FEVI después del trasplante y por ende se espera una mayor mortalidad.

El trasplante está contraindicado en pacientes con las siguientes características, a menos que se realice trasplante simultáneo corazón-riñón:

- Enfermedad multivaso que no sea posible revascularizar.

- Enfermedad valvular severa.

La mortalidad perioperatoria y postrasplante renal es muy elevada en pacientes con estas características ${ }^{4}$ por lo que están consideradas como contraindicaciones absolutas de trasplante renal. Sin embargo, en la medida de lo posible, se debe intentar realizar un trasplante simultáneo corazón-riñón en estos pacientes. Por esta misma razón, se recomienda realizar ecocardiograma en reposo en todos los pacientes que están siendo evaluados para trasplante renal (como se menciona en los puntos anteriores).

Pacientes con presión sistólica de la arteria pulmonar (PSAP) $>50 \mathrm{mmHg}$ requieren cateterismo de corazón derecho para determinar severidad y causa de la hipertensión pulmonar.

Entre $13-50 \%$ de los pacientes con ERCT tienen hipertensión pulmonar y 1-10\% tienen hipertensión pulmonar severa. ${ }^{24,25} \mathrm{La}$ incidencia de hipertensión pulmonar aumenta conforme se incrementa el tiempo en diálisis, de forma que cerca de $60 \%$ de los pacientes con más de dos años en diálisis tienen hipertensión pulmonar. ${ }^{24}$ Además, aquellos pacientes con hipertensión pulmonar previo al trasplante, especialmente aquéllos con PSAP > $50 \mathrm{mmHg}$, tienen mayor mortalidad después del trasplante..$^{24,25}$

Ya que la medición de PSAP por ecocardiografía no es exacta, los pacientes con hipertensión pulmonar con PSAP > $50 \mathrm{mmHg}$ por ecocardiografía requieren valoración con cateterismo de corazón derecho., ${ }^{4,25} \mathrm{De}$ esta forma se podrá clasificar a los pacientes en grupos según la etiología de la hipertensión pulmonar, lo cual también guía el tratamiento de la misma. La evaluación y tratamiento de hipertensión pulmonar debe ser dirigida por médicos especialistas en el tema. 
El trasplante renal está contraindicado en pacientes con hipertensión pulmonar no secundaria a sobrecarga de volumen (presión pulmonar media $>25 \mathrm{mmHg}$, presión de cuña $<15 \mathrm{mmHg}$, resistencia vascular pulmonar $>3$ $\mathrm{U})$ que no responden a tratamiento etiológico.

La mortalidad e incidencia de eventos cardiovasculares se eleva significativamente después del trasplante en pacientes con hipertensión pulmonar. ${ }^{4,24,25}$ Sin embargo, muchas causas de hipertensión pulmonar son reversibles. Es de particular importancia identificar a los pacientes que tienen hipertensión pulmonar por sobrecarga de volumen, o por insuficiencia cardiaca de alto gasto por alto flujo de fístula arteriovenosa, ya que estas causas son relativamente sencillas de revertir con disminución del peso seco o ligamiento de fístula arteriovenosa. ${ }^{25} \mathrm{De}$ igual forma, aquellos pacientes con hipertensión arterial pulmonar del grupo 1 (idiopática, enfermedad de tejido conectivo, etc.) se deben tratar con terapias dirigidas como sildenafil, bosentán, o prostaciclinas. ${ }^{25}$ Se debe valorar la respuesta al tratamiento etiológico, y de esto dependerá su candidatura a trasplante renal.

\section{EVALUACIÓN CARDIOVASCULAR DEL POTENCIAL DONADOR DE RIÑÓN}

La evaluación cardiovascular del donador debe ser similar a la evaluación cardiovascular para otras cirugías no cardiacas a excepción de la valoración de presión arterial.

Dado que el potencial donador de un injerto renal en general debe ser una persona sana sin problemas graves de salud, estos pacientes deben ser evaluados como la población general para estimar riesgos perioperatorios para cirugía no cardiaca. La excepción es la valoración de hipertensión arterial dado que la nefrectomía puede estar asociada con aumento de incidencia de hipertensión. ${ }^{26,27}$

La presión arterial (TA) del donador se debe medir por lo menos dos veces en diferentes ocasiones, y se debe mantener un bajo umbral para solicitar un monitoreo ambulatorio de presión arterial (MAPA) para descartar hipertensión de bata blanca o hipertensión enmascarada.

La presión arterial varía por múltiples situaciones, especialmente en estados de estrés, por lo que los dona- dores potenciales deben ser evaluados con medición de TA, por lo menos dos veces en ocasiones separadas. En caso de duda o sospecha de hipertensión de bata blanca o hipertensión enmascarada se deberá realizar MAPA.

Es importante notar que no está clara la definición de hipertensión arterial sistémica en este grupo de pacientes. La mayoría de los estudios realizados en donadores hasta la fecha han utilizado como definición de hipertensión > 140/90 mmHg en medición en consultorio médico, o >135/85 mmHg en MAPA o el uso de antihipertensivos. ${ }^{28}$ Sin embargo, las guías del American College of Cardiology y la American Heart Association de 2017 definen hipertensión en la población general $>130 / 80 \mathrm{mmHg} .^{29}$ Por el momento, no hay suficiente información para apoyar una definición de hipertensión arterial sistémica en el potencial donador.

La donación está contraindicada en pacientes que tienen evidencia de daño a órgano blanco causado por hipertensión arterial sistémica.

Uno de los órganos blanco de hipertensión arterial sistémica es el riñón. Por lo tanto, la evidencia de daño a algún órgano blanco aumenta la sospecha de daño renal por hipertensión. La evidencia de daño a órgano blanco incluye, pero no se limita retinopatía hipertensiva e hipertrofia de ventrículo izquierdo.

La donación está contraindicada en pacientes con hipertensión arterial sistémica descontrolada.

La hipertensión arterial sistémica puede causar daño renal, por lo que no se recomienda la donación renal en pacientes con hipertensión descontrolada. Sin embargo, en caso de controlarse con hábitos higiénicodietéticos o un antihipertensivo, el paciente se puede evaluar de nuevo como potencial donador.

Los potenciales donadores con hipertensión arterial sistémica controlada con un antihipertensivo sin evidencia de daño a órgano blanco pueden ser apropiados para donación, con las siguientes consideraciones:

- La decisión de aceptar como donador a un paciente con hipertensión debe individualizarse a cada paciente dependiendo de otros factores de riesgo (pacientes jóvenes vs. añosos, raza, obesidad, historia familiar de hipertensión, hiperlipidemia, 
tabaquismo, entre otras variantes) con base en el umbral de riesgo aceptado en cada institución.

- El paciente entiende que posterior a la donación puede requerir más medicamentos para controlar su TA, y que tener hipertensión antes de la donación probablemente está relacionado a un mayor riesgo de ERCT posterior a la donación.

Posterior a la donación renal, un gran porcentaje de pacientes desarrollan hipertensión arterial sistémica a largo plazo, principalmente aquéllos con más factores de riesgo (los cuales incluyen edad > 50 años, historia familiar de hipertensión, índice de masa corporal $>25 \mathrm{~kg} / \mathrm{m}^{2}$, tabaquismo, hiperlipidemia y mayor glucosa en ayunas). ${ }^{30}$ La raza hispana también aumenta el riesgo de desarrollar hipertensión postdonación. ${ }^{31}$ Sin embargo, no está claro que la donación per se sea la causa de la hipertensión arterial. En un metaanálisis de estudios realizados en la década de 1980-1990 se observó que aquellos pacientes que donaron un riñón comparados con personas sanas que no donaron, su presión arterial sistólica aumentó $6 \mathrm{mmHg}$ y la presión arterial diastólica aumentó 4 $\mathrm{mmHg}$ en 8-13 años de seguimiento. ${ }^{26}$ Sin embargo, un metaanálisis más reciente no mostró diferencias en presión arterial sistólica o mayor incidencia de hipertensión arterial sistémica posterior a la donación. ${ }^{27}$ No obstante, la hipertensión es una de las principales causas de enfermedad renal terminal a largo plazo en donadores renales, ${ }^{32}$ y la hipertensión postdonación se ha asociado a proteinuria y a disminución de la tasa de filtrado glomerular (TFG). ${ }^{30}$

$\mathrm{La}$ incidencia de desenlaces duros (muerte o enfermedad renal crónica terminal) en donadores hipertensos hasta el momento no ha sido definitoria. El único estudio realizado en México es el de Gracida y colaboradores, ${ }^{33}$ en el cual retrospectivamente se identificaron 16 donadores que tenían hipertensión arterial sistémica controlada con un antihipertensivo previo a la donación. Los donadores con hipertensión arterial sistémica previo al trasplante tenían una TFG ligeramente más baja que el grupo de donadores sin factores de riesgo (107.5 vs. $116 \mathrm{~mL} / \mathrm{min}$ ). En el postrasplante, con un seguimiento promedio de $80.7 \pm 32.58$ meses, los donadores hipertensos tuvieron una TFG de $78.1 \mathrm{~mL} /$ min, mientras que el grupo sin factores de riesgo tuvo TFG de $78.5 \mathrm{~mL} / \mathrm{min}$. Esta diferencia alcanzó significancia estadística, aunque la significancia clínica de una diferencia de $0.4 \mathrm{~mL} / \mathrm{min}$ en TFG es debatible. En un estudio coreano, Lee y su equipo ${ }^{34}$ reportaron desenlaces en seis donadores hipertensos controlados con $\leq 1$ antihipertensivo, con seguimiento de 89 meses en promedio. Posterior a la donación los pacientes requirieron uno o dos antihipertensivos para controlar su presión arterial. Cuatro de seis $(66 \%)$ donadores hipertensos presentaron una TFG estimada (TFGe) $<60 \mathrm{~mL} / \mathrm{min}$ en el último seguimiento, mientras que solamente $22 \%$ de los donadores no hipertensos tuvieron una TFGe $<60$ $\mathrm{mL} / \mathrm{min}(\mathrm{p}=0.02)$. Hipertensión previo a la donación se asoció con enfermedad renal crónica con un OR de 7.88 (IC95\% 1.14-54.4, p = 0.04). Sin embargo, es importante notar que estos dos estudios están limitados por el pequeño número de donadores hipertensos y por su diseño retrospectivo. ${ }^{33,34}$ En un estudio prospectivo, Textor y colaboradores ${ }^{35}$ identificaron 24 donadores hipertensos (con medición de TA en el consultorio y con MAPA) y se les dio seguimiento por un año después de donar. En un análisis multivariado se observó que la hipertensión predonación no se asoció con menor TFG o con proteinuria postdonación. La limitación más importante de este estudio es el corto tiempo de seguimiento. El estudio de Sanchez y colaboradores ${ }^{30}$ reportó desenlaces en 96 donadores hipertensos al momento de la donación comparados con donadores no hipertensos, y encontró que no había diferencias en desarrollo de proteinuria, disminución de la TFG, enfermedad renal crónica terminal o muerte posterior a la donación. El estudio con mayor población que se ha hecho hasta el momento es el de Segev y su equipo, ${ }^{36}$ en el cual se analizó la base de datos de UNOS en Estados Unidos y se incluyeron 80,347 donadores (545 con hipertensión previo a la donación), los cuales fueron comparados con 9,364 controles (pacientes sanos no hipertensos del estudio NHANES III que podrían donar, pero no lo hicieron). En este estudio se observó que el diagnóstico de hipertensión predonación no se asoció con mortalidad a largo plazo. Sin embargo, la presión arterial sistólica pretrasplante $\geq 140 \mathrm{mmHg}$ se asoció con mayor mortalidad a 12 años (HR 3.3; IC95\% 1.1-9.7) en comparación con < $120 \mathrm{mmHg}$. Este estudio no analizó desenlaces renales.

En conjunto no está del todo claro que el diagnóstico de hipertensión previo a la donación se asocie a desenlaces adversos en el periodo postdonación. Sin embargo, tampoco hay suficiente evidencia para declarar de forma definitiva que la donación en pacientes hipertensos es completamente inocua. Pero parece ser que el riesgo absoluto de desenlaces adversos en estos pacientes es relativamente bajo. Es por esto que recomendamos individualizar la decisión de aceptar a un donador hipertenso tomando en cuenta otros factores. Especialmente importante es tomar en cuenta la 
edad. Dado que la esperanza de vida de un paciente joven es mucho mayor que un paciente añoso, la incidencia de desenlaces adversos a largo plazo después de la donación es mayor en los pacientes jóvenes. De esta forma, consideramos inapropiado aceptar como donador a pacientes hipertensos jóvenes. De igual forma se deben tomar en cuenta otros factores de riesgo incluyendo historia familiar de hipertensión, índice de masa corporal $>25 \mathrm{~kg} / \mathrm{m}^{2}$, tabaquismo, hiperlipidemia y glucosa anormal en ayunas.

Es imprescindible y de extrema importancia obtener un consentimiento del donador hipertenso donde el proveedor de salud se cerciore de que el paciente esté bien informado y que entienda los riesgos de la donación.

Es importante hacer notar que la evidencia para esta recomendación es baja. Se necesitan nuevos y mejores estudios que evalúen la incidencia de efectos adversos posterior a la donación en pacientes hipertensos.

\section{REFERENCIAS}

1. Nakano T, Ninomiya T, Sumiyoshi S et al. Association of kidney function with coronary atherosclerosis and calcification in autopsy samples from Japanese elders: the Hisayama study. Am J Kidney Dis. 2010; 55: 21-30.

2. Chonchol M, Whittle J, Desbien A, Orner MB, Petersen LA, Kressin NR. Chronic kidney disease is associated with angiographic coronary artery disease. Am J Nephrol. 2008; 28 (2): 354-360.

3. Ramphul R, Fernández M, Firoozi S, Kaski JC, Sharma R, Banerjee D. Assessing cardiovascular risk in chronic kidney disease patients prior to kidney transplantation: clinical usefulness of a standardised cardiovascular assessment protocol. BMC Nephrol. 2018; 19: 2.

4. Lentine KL, Costa SP, Weir MR et al. Cardiac disease evaluation and management among kidney and liver transplantation candidates: a scientific statement from the American Heart Association and the American College of Cardiology Foundation. J Am Coll Cardiol. 2012; 60: 434-480.

5. Lentine KL, Brennan DC, Schnitzler MA. Incidence and predictors of myocardial infarction after kidney transplantation. J Am Soc Nephrol. 2005; 16: 496-506.

6. Kasiske BL, Maclean JR, Snyder JJ. Acute myocardial infarction and kidney transplantation. J Am Soc Nephrol. 2006; 17: 900907.

7. John R, Lietz K, Huddleston $\mathrm{S}$ et al. Perioperative outcomes of cardiac surgery in kidney and kidney-pancreas transplant recipients. J Thorac Cardiovasc Surg. 2007; 133: 1212-1219.

8. Zhang L, Garcia JM, Hill PC, Haile E, Light JA, Corso PJ. Cardiac surgery in renal transplant recipients: experience from Washington Hospital Center. Ann Thorac Surg. 2006; 81: 13791384.

9. Mitruka SN, Griffith BP, Kormos RL et al. Cardiac operations in solid-organ transplant recipients. Ann Thorac Surg. 1997; 64: 1270-1278.

10. Massad $\mathrm{M}$, Kpodonu $\mathrm{J}$, Lee $\mathrm{J}$ et al. Outcome of coronary artery bypass operations in patients with renal insufficiency with and without renal transplantation. Chest. 2005; 128: 855-862.
11. Cohen-Bucay A, Gordon CE, Francis JM. Non-immunological complications following kidney transplantation. F1000Res. 2019; 8.

12. Ducloux D, Kazory A, Chalopin JM. Predicting coronary heart disease in renal transplant recipients: a prospective study. Kidney Int. 2004; 66: 441-447.

13. Kiberd B, Panek R. Cardiovascular outcomes in the outpatient kidney transplant clinic: the Framingham risk score revisited. Clin J Am Soc Nephrol. 2008; 3: 822-828.

14. Wang LW, Fahim MA, Hayen A et al. Cardiac testing for coronary artery disease in potential kidney transplant recipients. Cochrane Database Syst Rev. 2011: CD008691.

15. Rosario MA, Lima JJ, Parga JR et al. Coronary calcium score as predictor of stenosis and events in pretransplant renal chronic failure. Arq Bras Cardiol. 2010; 94: 236-243, 52-60, 9-47.

16. Winther S, Bottcher M, Jorgensen HS et al. Coronary calcium score may replace cardiovascular risk factors as primary risk stratification tool before kidney transplantation. Transplantation. 2016; 100: 2177-2187.

17. Haydar AA, Hujairi NM, Covic AA, Pereira D, Rubens M, Goldsmith DJ. Coronary artery calcification is related to coronary atherosclerosis in chronic renal disease patients: a study comparing EBCT-generated coronary artery calcium scores and coronary angiography. Nephrol Dial Transplant. 2004; 19: 23072312.

18. Sharples EJ, Pereira D, Summers $S$ et al. Coronary artery calcification measured with electron-beam computerized tomography correlates poorly with coronary artery angiography in dialysis patients. Am J Kidney Dis. 2004; 43 (2): 313-319.

19. lio K, Nagasawa $Y$, Kimura $T$ et al. Assessment of coronary stenosis by a 16 -slice MDCT scanner in asymptomatic diabetic patients starting dialysis therapy. Nephron Clin Pract. 2008; 109: c72-79.

20. Wang LW, Masson P, Turner RM et al. Prognostic value of cardiac tests in potential kidney transplant recipients: a systematic review. Transplantation. 2015; 99: 731-745.

21. Joki N, Hase H, Saijyo T et al. Combined assessment of cardiac systolic dysfunction and coronary atherosclerosis used to predict future cardiac deaths after starting hemodialysis. Am J Nephrol. 2003; 23 (6): 458-465.

22. Yamada $\mathrm{S}$, Ishii $\mathrm{H}$, Takahashi $\mathrm{H}$ et al. Prognostic value of reduced left ventricular ejection fraction at start of hemodialysis therapy on cardiovascular and all-cause mortality in end-stage renal disease patients. Clin J Am Soc Nephrol. 2010; 5: 17931798.

23. Wali RK, Wang GS, Gottlieb SS et al. Effect of kidney transplantation on left ventricular systolic dysfunction and congestive heart failure in patients with end-stage renal disease. J Am Coll Cardiol. 2005; 45: 1051-1060.

24. Issa N, Krowka MJ, Griffin MD, Hickson LJ, Stegall MD, Cosio FG. Pulmonary hypertension is associated with reduced patient survival after kidney transplantation. Transplantation. 2008; 86: 1384-1388.

25. Lentine KL, Villines TC, Axelrod D, Kaviratne S, Weir MR, Costa $\mathrm{SP}$. Evaluation and management of pulmonary hypertension in kidney transplant candidates and recipients: concepts and controversies. Transplantation. 2017; 101: 166-181.

26. Boudville N, Prasad GV, Knoll G et al. Meta-analysis: risk for hypertension in living kidney donors. Ann Intern Med. 2006; 145: 185-196.

27. O'Keeffe LM, Ramond A, Oliver-Williams $C$ et al. Mid- and longterm health risks in living kidney donors: a systematic review and meta-analysis. Ann Intern Med. 2018; 168: 276-284. 
28. Young A, Storsley L, Garg AX et al. Health outcomes for living kidney donors with isolated medical abnormalities: a systematic review. Am J Transplant. 2008; 8: 1878-1890.

29. Whelton PK, Carey RM, Aronow WS et al. 2017 ACC/AHA/AAPA/ ABC/ACPM/AGS/APhA/ASH/ASPC/NMA/PCNA guideline for the prevention, detection, evaluation, and management of high blood pressure in adults: a report of the American College of Cardiology/American Heart Association Task Force on Clinical Practice Guidelines. Hypertension. 2018; 71: e13-e115.

30. Sanchez OA, Ferrara LK, Rein S, Berglund D, Matas AJ, Ibrahim $\mathrm{HN}$. Hypertension after kidney donation: Incidence, predictors, and correlates. Am J Transplant. 2018; 18: 2534-2543.

31. Lentine $\mathrm{KL}$, Schnitzler MA, Xiao $\mathrm{H}$ et al. Racial variation in medical outcomes among living kidney donors. N Engl J Med. 2010; 363: 724-732.

32. Anjum S, Muzaale AD, Massie AB et al. Patterns of end-stage renal disease caused by diabetes, hypertension, and glomerulonephritis in live kidney donors. Am J Transplant. 2016; 16: 3540-3547.
33. Gracida C, Espinoza R, Cedillo U, Cancino J. Kidney transplantation with living donors. Transplantation Proceedings. 2003; 35: 946-947.

34. Lee JH, Kim SC, Han DJ et al. Risk factors for MDRD-GFR of less than $60 \mathrm{~mL} / \mathrm{min}$ per $1.73 \mathrm{~m}^{2}$ in former kidney donors. Nephrology (Carlton). 2007; 12: 600-606.

35. Textor SC, Taler SJ, Driscoll $\mathrm{N}$ et al. Blood pressure and renal function after kidney donation from hypertensive living donors. Transplantation. 2004; 78: 276-282.

36. Segev DL, Muzaale AD, Caffo BS et al. Perioperative mortality and long-term survival following live kidney donation. JAMA. 2010; 303: 959-966.

Correspondencia:

Dr. Abraham Cohen-Bucay

E-mail: acohenbucay@mgh.harvard.edu 